\title{
Nível de Arraçoamento para Alevinos de Lambari-do-Rabo-Amarelo (Astyanax bimaculatus)
}

\author{
Fábio Meurer ${ }^{1}$, Carmino Hayashi ${ }^{2}$, Wilson Rogério Boscolo ${ }^{3}$, Lúcio Barboza Kavata ${ }^{4}$, Carlos \\ Henrique Figueiredo Lacerda 4
}

\begin{abstract}
RESUMO - Este trabalho foi realizado com o objetivo de determinar o nível ideal de arraçoamento, em porcentagem do peso vivo, para alevinos de lambari-do-rabo-amarelo (Astyanax bimaculatus). Foram utilizados 240 alevinos de lambari com peso vivo de $0,52 \pm 0,05 \mathrm{~g}$, distribuídos em 24 hapas, em um delineamento em blocos inteiramente casualizados, com seis tratamentos e quatro repetições. Cada bloco correspondeu a uma caixa de fibrocimento com capacidade para $1.000 \mathrm{~L}$, com seis hapas, cada uma com 10 alevinos. Cada hapa foi considerada uma unidade experimental. Os tratamentos consistiram dos níveis de arraçoamento de 1, 4, 7, 10, 13 e 16\% do peso vivo dos animais em ração ( $30 \%$ proteína digestível e $3.000 \mathrm{kcal} / \mathrm{kg}$ de energia digestível). Os parâmetros físico-químicos médios aferidos da água foram $24,5^{\circ} \mathrm{C} ; 25,1^{\circ} \mathrm{C} ; 8,4 \mathrm{mg} / \mathrm{L} ; 7,9$ e $160,9 \mu \mathrm{S} / \mathrm{cm}$, respectivamente, para temperaturas matutina e vespertina, oxigênio dissolvido, $\mathrm{pH}$ e condutividade. Os resultados indicam que o melhor nível de arraçoamento para alevinos de lambari do rabo amarelo à temperatura média de $25^{\circ} \mathrm{C}$ é de $11,5 \%$ do peso vivo.
\end{abstract}

Palavras-chave: alimentação, Astyanax bimaculatus, desempenho, lambari, piscicultura

\section{Feeding Level for Yellow Tail Lambari (Astyanax bimaculatus) Fingerlings}

\begin{abstract}
The current work aimed to evaluate the ideal diet level for yellow tail lambari (Astyanax bimaculatus) fingerlings on body weight percentage. Two hundred and forty fingerlings averaging initial weight of $0.52 \pm 0.05 \mathrm{~g}$ were allotted to 24 hapas according to a completely randomized blocks with six treatments and four replicates. A $1000 \mathrm{~L}$-tank with 6 hapas was considered the block. A hapa with 10 fingerlings was considered the experimental unit. The treatments consisted of increasing feeding levels: 1, 4, 7 10, 13, and 16\% of body weight $(30 \%$ of digestible protein and $3,000 \mathrm{kcal} / \mathrm{kg}$ of digestible energy). The observed water parameters were as follows: $24.5^{\circ} \mathrm{C}, 25.1^{\circ} \mathrm{C}, 8.4 \mathrm{mg} / \mathrm{L}, 7.9$ e $160.9 \mu \mathrm{S} / \mathrm{cm}$, respectively, for morning and afternoon temperature, dissolved oxygen, $\mathrm{pH}$, and electric conductivity. The results showed that the best level of feeding for yellow tail lambari fingerlings on average water temperature of $25^{\circ} \mathrm{C}$ was of $11.5 \%$ of body weight.
\end{abstract}

Key Words: aquaculture, Astyanax bimaculatus, lambari, performance

\section{Introdução}

A piscicultura é um ramo da zootecnia atualmente em evidência no Brasil, fato relacionado à grande aptidão do país para este tipo de atividade, do clima adequado e da grande disponibilidade de água, acrescido pela ótima qualidade nutricional desse peixe. Entre as espécies mais cultivadas, estão as de origem exótica, como a tilápia e as carpas. Entretanto, dentro da ampla diversidade da fauna aquática brasileira, são encontradas espécies que se adequam perfeitamente ao cultivo, como o pacu, o tambaqui, o piavuçu, entre outros.

Olambari-do-rabo-amarelo(Astyanaxbimaculatus) é uma espécie nativa, de pequeno porte, que aceita alimentação artificial com bastante facilidade e apresenta bom potencial para a aquicultura, pois é bastante apreciado como petisco, além de ser muito requisitado como isca para a pesca esportiva. Alguns aspectos de sua reprodução já são dominados e, na literatura, há estudos envolvendo nutrição (Hayashi et al., 1999a; Soares et al., 1999) e densidade (Vilela \& Hayashi, 2001).

Em comparação aos animais domésticos explorados comercialmente, como suínos, aves e bovinos, a disponibilidade de informações sobre os aspectos de manejo e nutrição para a piscicultura, mesmo em espécies mais exploradas, como o catfish americano, salmonídeos, carpas e tilápias, é muito menor. Em relação a espécies nativas, o número de informa-

\footnotetext{
1 Zootecnista, Dr., Professor dos cursos de Agronomia, Enfermagem e Medicina Veterinária do CCTP da Pontifícia Universidade Católica do Paraná - Campus Toledo. Av. da União 500, Toledo-PR, CEP: 85902-532 (fabio.meurer@pucpr.br; fabio_meurer@yahoo.com.br).

${ }^{2}$ Biólogo, Dr., Pesquisador da Centro Avançado de Pesquisa Tecnológica e do Agronegócio de Pescado Continental, APTA/SAA, São José do Rio Preto, SP. Pesquisador do CNPq.

3 Zootecnista, Dr., Prof. de Eng. de Pesca/UNIOESTE.

4 Zootecnista.
} 
ções é bastante inferior, o que dificulta o cultivo racional destas espécies, podendo levar ao baixo retorno econômico da atividade.

Para estudos relacionados à nutrição, são necessários conhecimentos básicos de biologia e manejo dos peixes. Um dos fatores importantes a ser considerado é o nível de arraçoamento mais adequado para o correto desenvolvimento da espécie. O nível de arraçoamento da espécie varia principalmente em função da temperatura da água e da fase de crescimento, sendo que o nível de oxigênio dissolvido e amônia também vão influenciar a quantidade de ração consumida (Hidalgo et al., 1987; Santiago et al., 1987; Storebakken \& Austreng, 1987; Lim, 1995; Kubitza, 1997). A variação dos níveis de arraçoamento pode ter efeito sobre o crescimento, metabolismo, digestibilidade, composição da carcaça e excreção de amônia (Johansson et al., 2000; Verbeeten et al., 1999; Fernández et al., 1998; Shimeno et al., 1997).

O nível de arraçoamento pode influenciar diretamente o retorno econômico no cultivo de peixes, pois o arraçoamento aquém das necessidades fisiológicas pode resultar no baixo desempenho animal, o que aumenta o tempo de cultivo para a obtenção do peso de mercado. O excesso de ração fornecida pode acarretar perda de ração, fato que, além da perda monetária relacionada ao desperdício de ração, pode determinar o decréscimo na qualidade de água do viveiro e maior impacto ambiental.

A qualidade da água do viveiro está diretamente relacionada ao desempenho e à sobrevivência do peixe, pois níveis elevados de compostos nitrogenados, como é o caso da amônia e do nitrito, podem causar problemas desde a diminuição do desempenho até a mortalidade por intoxicação (Ostrensky \& Boeger, 1998; Kubitza, 2000). Outro problema com a qualidade de água relacionado ao excesso de arraçoamento é a diminuição dos níveis de oxigênio dissolvido durante a madrugada, em função do excesso de eutrofização, podendo causar a diminuição do desempenho, susceptibilidade a doenças e mortalidade.

O objetivo neste experimento foi testar diferentes níveis de arraçoamento sobre os parâmetros de desempenho e sobrevivência de alevinos de lambari-dorabo-amarelo (Astyanax bimaculatus).

\section{Material e Métodos}

O experimento foi realizado no Laboratório de Aquicultura, do Departamento de Biologia da Universidade Estadual de Maringá, durante o período de 6 de abril a 6 de maio de 2001. Foram utilizados 240 alevinos de lambari-do-rabo-amarelo (A. bimaculatus) com peso inicial de $0,52 \pm 0,05 \mathrm{~g}$, distribuídos em 24 hapas (tanque-rede de sombrite de pequeno volume), em um delineamento em blocos inteiramente casualizados, com seis tratamentos e quatro repetições, em que cada bloco correspondia a um tanque de fibrocimento com capacidade para $1.000 \mathrm{~L}$ contendo seis hapas. Cada hapa com dez alevinos foi considerada uma unidade experimental.

As hapas possuíam aeração constante, por meio de pedras porosas ligadas por mangueiras e um regulador de ar, num soprador; a temperatura foi mantida por dois aquecedores de $200 \mathrm{~W}$ em cada tanque. Os tanques contavam com taxa de renovação diária de $20 \%$ do volume de água. A temperatura da água dos tanques foi aferida no início da manhã e ao final da tarde; o oxigênio dissolvido, o $\mathrm{pH}$ e a condutividade foram aferidos uma vez por semana, durante o período da manhã, após a qual era efetuada a sifonagem para retirada das fezes. Diariamente, antes da primeira alimentação, era efetuado a sifonagem dos tanques com níveis de arraçoamento de 13 e $16 \%$ de peso vivo, com o intuito de se retirar as sobras de ração do dia anterior, procedimento não realizado nos demais tratamentos.

Para a fabricação das rações (Tabela 1), os alimentos foram moídos, misturados e peletizados de acordo com Hayashi et al. (1999b). Os peletes foram moídos para adequação dos mesmos ao tamanho da boca dos alevinos. A freqüência de arraçoamento dos alevinos foi feita de acordo com Hayashi et al. (2004), quatro vezes ao dia às $8,11,14 \mathrm{e} 17 \mathrm{~h}$.

Os tratamentos constituíram-se de diferentes níveis de fornecimento de ração $(1,4,7,10,13$ e $16 \%$ do peso vivo) aos alevinos de lambari. Como o crescimento dos peixes, especialmente nesta fase, é bastante acentuado e considerando-se que o foco do experimento foi justamente o estudo da quantidade de ração fornecida e o peso vivo do peixe, foi importante a manutenção da constância do nível de arraçoamento dos alevinos durante o período experimental. Portanto, 
Tabela 1 - Composições percentual e bromatológica da ração experimental Table 1 - Ingredient and chemical compositions of experimental diet (\%)

\begin{tabular}{|c|c|c|c|}
\hline $\begin{array}{l}\text { Alimento } \\
\text { Feedstuff }\end{array}$ & $(\%)$ & $\begin{array}{l}\text { Composição } \\
\text { Composition }\end{array}$ & \\
\hline Farelo de soja ${ }^{1}$ & 49,92 & Proteína digestível, \% & 30,0 \\
\hline Soybean meal & & Digestible protein & \\
\hline Milho moído ${ }^{1}$ & 35,96 & Energia digestível (kcal $\backslash \mathrm{kg})$ & 3000 \\
\hline Maize & & Digestible energy (kcal $\mathrm{kg}$ ) & \\
\hline Proteinoso milho ${ }^{2}$ & 9,06 & Amido, $\%$ & 29,03 \\
\hline Maize gluten meal & & Starch & \\
\hline Óleo de soja ${ }^{1}$ & 0,63 & Gordura, $\%$ & 4,00 \\
\hline Soybean oil & & Fat & \\
\hline Fosfato bicálcico ${ }^{3}$ & 2,41 & Fibra bruta, $\%$ & 3,22 \\
\hline Dicalcium phosphate & & Crude fiber & \\
\hline Calcário $^{3}$ & 0,89 & Lisina, $\%$ & 1,60 \\
\hline Limestone & & Lysine & \\
\hline Sal & 0,50 & Metionina + Cistina (\%) & 0,90 \\
\hline Salt & & Methionine + cystine & \\
\hline Suplemento vit-mineral ${ }^{4}$ & 0,50 & Fósforo total, $\%$ & 0,80 \\
\hline Vitamin and mineral premix & & Total phosphorus & \\
\hline DL-metionina 99 & 0,10 & Cálcio,\% & 1,00 \\
\hline DL-methionine 99 & & Calcium & \\
\hline BHT & 0,02 & Linoléico \% & 1,51 \\
\hline & & Linoleic & \\
\hline
\end{tabular}

${ }^{1}$ De acordo com os dados de análise bromatológica e digestibilidade de Boscolo et al. (2002).

${ }^{1}$ According to the digestibility and chemical values from Boscolo et al. (2002).

${ }^{2}$ De acordo com os dados de análise bromatológica e digestibilidade de Meurer et al. (2003).

${ }^{2}$ According to the digestibility and chemical values from Meurer et al. (2003).

${ }^{3}$ De acordo com os dados de Rostagno et al. (2000).

${ }^{3}$ According to the chemical values from Rostagno et al. (2000).

${ }^{4}$ Níveis de garantia por quilograma do produto: Vit. A,1.200.000 UI; Vit. D3, $200.000 \mathrm{UI}$; Vit. E, $12.000 \mathrm{mg}$; Vit. K3, 2.400 mg; Vit. B1, $4.800 \mathrm{mg}$; Vit. B2, $4.800 \mathrm{mg}$; Vit. B6, $4.000 \mathrm{mg}$; Vit. B12, $4.800 \mathrm{mg}$; Ác. fólico, $1.200 \mathrm{mg}$; Pantotenato Ca, $12.000 \mathrm{mg}$; Vit. C, 48.000 mg; Biotina, 48 mg; Colina, 65.000 mg; Niacina, 24.000 mg; Ferro, 10.000 mg; Cobre, 6.000 mg; Manganês, 4.000 mg; Zinco, 6.000 mg; lodo, $20 \mathrm{mg}$; Cobalto, $2 \mathrm{mg}$; Selênio, $20 \mathrm{mg}$.

${ }^{4}$ Guarantee levels by kilogram of product: Vit. A, 1,200,000 UI; Vit. D, 200,000 Ul; Vit. E, 12,000 mg; Vit. K3, 2,400 mg; Vit. B1, 4,800 mg; Vit. B2, 4,800 mg; Vit. B6, 4,000 mg; Vit. B12, 4,800 mg; Folic acid, 1,200 mg; Ca pantotenate, 12,000 mg; Vit. C, 48,000 mg; Biotin, $48 \mathrm{mg} ;$ Colin, 65,000 mg; Niacin, 24,000 mg; Fe, 10,000 mg; Cu, 6,000 mg; Mn, 4,000 mg; Zn, 6,000 mg; l, 20 mg; Co, 2 mg; Se, 20 mg.

$3 \mathrm{kcal} / \mathrm{kg}$.

a quantidade de ração fornecida era acrescida em $5 \%$, diariamente, para a adequação entre o fornecimento da ração e o peso vivo dos alevinos.

Em decorrência da preocupação na manutenção da constância da porcentagem do arraçoamento, também foram feitas pesagens de cada unidade experi-

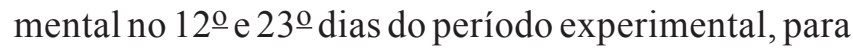
aferição e reajuste da quantidade de ração fornecida, em função do crescimento diferenciado influenciado por cada tratamento.

Ao final do experimento, os peixes de cada unidade experimental foram pesados e medidos, para avaliação das variáveis de peso final total, peso final médio, ganho de peso médio, comprimento final médio, conversão alimentar aparente e sobrevivência. Os valores dos parâmetros físico-químicos da água e do desempenho final dos animais, bem como os das pesagens intermediárias, foram então submetidos à análise de variância a 5\% de probabilidade e, havendo diferença estatística, foram submetidos à análise de regressão, pelo programa computacional SAEG - Sistema de Análises Estatísticas e Genéticas (UFV, 1997).

\section{Resultados e Discussão}

Os valores médios para as temperaturas matutina e vespertina foram, respectivamente, de $24,5^{\circ} \mathrm{C} ; 25,1^{\circ} \mathrm{C}$. Os resultados médios das variáveis físico-químicas da água foram $8,4 \mathrm{mg} / \mathrm{L} ; 7,9$ e $160,9 \mu \mathrm{S} / \mathrm{cm}$, respectivamente, para oxigênio dissolvido, pH e condutividade elétrica. Não houve diferenças estatísticas entre os tratamentos nos parâmetros de qualidade de água e de temperatura 
mensurados e, de acordo com Sipaúba-Tavares (1995), esses valores atenderam os padrões recomendados para a piscicultura.

Os valores do peso total, peso médio e ganho de peso médio das pesagens intermediárias $\left(12^{\circ} \mathrm{o}\right.$ e $23^{\circ}$ dia de experimento) dos alevinos de lambari submetidos à variação na freqüência de arraçoamento encontram-se na Tabela 2 .
Os valores médios do peso inicial, do peso final do lote, do comprimento final médio, do peso final, do ganho de peso, da conversão alimentar e da sobrevivência dos alevinos de lambari (A. bimaculatus) submetidos aos diversos níveis de arraçoamento encontram-se na Tabela 3.

$O$ peso médio apresentado pelos alevinos de lambari demonstrou ao $12 \underline{\text { o }}$ dia efeito linear $(\mathrm{P}<0,01)$

Tabela 2 - Parâmetros de desempenho, aos 12으 e 23으 dias de experimento, dos alevinos de lambari submetidos à variação no nível de arraçoamento

Table 2 - Performance parameters, at $12^{\text {th }}$ and $23^{\text {rd }}$ experimental days, of lambari fingerlings on different feeding levels

\begin{tabular}{|c|c|c|c|c|c|c|c|}
\hline \multirow[t]{2}{*}{$\begin{array}{l}\text { Parâmetro mensurado }(\mathrm{g}) \\
\text { Measured parameter }(g)\end{array}$} & \multicolumn{6}{|c|}{$\begin{array}{c}\text { Nível de arraçoamento } \\
\text { Feedinglevel }\end{array}$} & \multirow[t]{2}{*}{$\mathrm{CV}(\%)$} \\
\hline & 1 & 4 & 7 & 10 & 13 & 16 & \\
\hline $\begin{array}{l}\text { Peso médio aos } 12 \text { dias }^{1} \\
\text { Average weight at } 12^{\text {th }} \text { day }{ }^{1}\end{array}$ & 0,56 & 0,71 & 0,79 & 0,87 & 0,84 & 0,95 & 10,58 \\
\hline $\begin{array}{l}\text { Peso médio aos } 23 \text { dias }^{2} \\
\text { Average weight at } 23^{\text {rd }} \text { day }\end{array}$ & 0,69 & 1,23 & 1,41 & 1,58 & 1,55 & 1,57 & 12,47 \\
\hline $\begin{array}{l}\text { Ganho de peso médio aos } 12 \text { dias }^{3} \\
\text { Average weight gain at } 12^{\text {th }} \text { day }^{3}\end{array}$ & 0,03 & 0,19 & 0,26 & 0,35 & 0,31 & 0,42 & 18,42 \\
\hline $\begin{array}{l}\text { Ganho de peso médio aos } 23 \text { dias }^{4} \\
\text { Average weight gain at } 23^{\text {rd }} \text { day }^{4}\end{array}$ & 0,16 & 0,71 & 0,88 & 1,06 & 1,02 & 1,04 & 18,64 \\
\hline
\end{tabular}

${ }^{1}$ Efeito linear (Linear effect): $\hat{Y}=0,59164+0,0230423 x, r^{2}=0,89$.

${ }^{2}$ Efeito quadrático (Quadratic effect): $\hat{Y}=0,57514+0,168783 x-0,00683148 x^{2}, r^{2}=0,97$.

${ }^{3}$ Efeito quadrático (Quadratic effect): $\hat{Y}=0,00326082+0,0463569 x-0,00139385 x^{2}, r^{2}=0,94$.

${ }^{3}$ Efeito quadrático (Quadratic effect): $\hat{Y}=0,0488247+0,169161 x-0,00687612 x^{2}, r^{2}=0,97$.

Tabela 3 - Resultados finais do desempenho e sobrevivência de alevinos de lambari (A. bimaculatus) submetidos a diferentes níveis de arraçoamento

Table 3 - Final performance parameters of lambari fingerlings on different feeding levels

\begin{tabular}{|c|c|c|c|c|c|c|c|}
\hline \multirow[t]{2}{*}{$\begin{array}{l}\text { Parâmetro mensurado }(\mathrm{g}) \\
\text { Measured parameter }(g)\end{array}$} & \multicolumn{6}{|c|}{$\begin{array}{c}\text { Nível de arraçoamento } \\
\text { Feeding level }\end{array}$} & \multirow[t]{2}{*}{$C V(\%)$} \\
\hline & 1 & 4 & 7 & 10 & 13 & 16 & \\
\hline $\begin{array}{l}\text { Peso inicial } \\
\text { Initial weight }\end{array}$ & 0,52 & 0,52 & 0,53 & 0,52 & 0,53 & 0,53 & 1,38 \\
\hline $\begin{array}{l}\text { Biomassa final }^{1} \\
\text { Final biomass }\end{array}$ & 7,22 & 14,30 & 15,77 & 17,80 & 16,90 & 16,60 & 11,68 \\
\hline $\begin{array}{l}\text { Comprimento final médio } \\
\text { Mean final length }\end{array}$ & 4,00 & 4,87 & 5,00 & 5,27 & 5,07 & 5,07 & 8,43 \\
\hline $\begin{array}{l}\text { Peso final médio }{ }^{3} \\
\text { Mean final weight }\end{array}$ & 0,74 & 1,43 & 1,57 & 1,87 & 1,69 & 1,70 & 11,16 \\
\hline $\begin{array}{l}\text { Ganho de peso } \\
\text { Weight gain }\end{array}$ & 0,22 & 0,91 & 1,04 & 1,35 & 1,16 & 1,17 & 17,73 \\
\hline $\begin{array}{l}\text { Conversão alimentar } \\
\text { Food conversion }\end{array}$ & 1,12 & 1,14 & 1,99 & 2,50 & 3,47 & 4,49 & 17,58 \\
\hline $\begin{array}{l}\text { Sobrevivência (\%) } \\
\text { Survival }\end{array}$ & 97,5 & 100,0 & 100,0 & 95,0 & 100,0 & 97,50 & 4,15 \\
\hline
\end{tabular}

1 Efeito quadrático (Quadratic effect): $\hat{Y}=5,94402+2,1042 x-0,092014 x^{2}, r^{2}=0,80$.

2 Efeito quadrático (Quadratic effect): $\hat{Y}=3,85778+0,250952 x-0,0112765 x^{2}, r^{2}=0,92$.

3 Efeito quadrático (Quadratic effect): $\hat{Y}=0,597034+0,214569 x-0,00932212 x^{2}, r^{2}=0,81$.

4 Efeito quadrático (Quadratic effect): $\hat{Y}=0,0707187+0,21496 x-0,00936673 x^{2}, r^{2}=0,80$.

5 Efeito linear (Linear effect) $\hat{Y}=0,479691+0,232095 x, r^{2}=0,87$. 
dos tratamentos em função do aumento no nível de arraçoamento. No 23ํㅡ dia, o mesmo parâmetro de desempenho apresentou efeito quadrático, em função do aumento no nível de arraçoamento, com o nível de arraçoamento para o melhor peso médio em $12,3 \%$ do peso vivo. Para o ganho de peso, tanto ao $12 \mathrm{o}$ quanto ao $23^{\circ}$ dias, houve comportamento quadrático com o aumento do nível de arraçoamento, com os respectivos pontos de máximo ganho de peso em 16 e 12,3\% de arraçoamento em função do peso vivo.

Os resultados de peso final do lote, peso final médio, ganho de peso e comprimento final médio apresentaram efeito significativo do nível de arraçoamento $(\mathrm{P}<0,01)$. Esses parâmetros observados tiveram efeito quadrático em relação ao nível de arraçoamento, com resultado de ponto de máxima de 11,$4 ; 11,5 ; 11,5$ e $11,1 \%$ do peso vivo em ração para alevinos de lambari (A. bimaculatus), respectivamente, para peso final do lote, peso final médio, ganho de peso e comprimento final médio.

A conversão alimentar dos alevinos apresentou aumento linear $(\mathrm{P}<0,01)$ em relação ao aumento do nível de arraçoamento, o que demonstra a piora do aproveitamento da ração conforme se aumenta o nível de arraçoamento, fato confirmado pelas sobra de ração nos dois tratamentos com níveis mais altos de arraçoamento. Não houve diferenças significativas $(\mathrm{P}>0,05)$ da sobrevivência dos alevinos em relação aos níveis de arraçoamento empregados.

Os resultados de ganho de peso e conversão alimentar estão de acordo com os valores apresentados por Santiago et al. (1987), para larvas de tilápia-do-nilo (Oreochromis niloticus), e Storebakken \& Austreng (1987), para larvas e alevinos de salmão do Atlântico (Salmo salar).

Shimeno et al. (1997) encontraram diferenças quanto aos valores de crescimento e eficiência alimentar para juvenis de carpa comum (Cyprinus carpio). Entretanto, esses autores não utilizaram níveis de arraçoamento que excediam a saciedade; além do tratamento sem alimentação, também não utilizaram análise de regressão nos dados. À medida em que se elevavam os níveis de arraçoamento até cerca de $70 \%$ do valor de saciedade, o ganho de peso médio aumentava. Para alevinos de greenback flounder (Rhombosolea tapirina), Verbeeten et al. (1999) encontraram diferença estatística $(\mathrm{P}<0,05)$, pelo teste Tukey, em relação aos valores de crescimento diário. Esses autores utilizaram três níveis de arraçoamento (1, 2 e 3\%), sendo que o último nível foi o valor de saciedade. Porém, para a eficiência alimentar, não houve diferença estatística $(\mathrm{P}>0,05)$ entre os tratamentos.

Nota-se que, em ambos os experimentos supracitados, à medida em que se aumentava o nível de arraçoamento, havia melhoria no crescimento dos animais, de modo semelhante ao ocorrido neste estudo com os valores médios de ganho de peso e peso final até o tratamento com nível de arraçoamento de $10 \%$, valor mais próximo ao de melhor desempenho para estas características, calculado por meio da equação de regressão quadrática ajustada aos valores encontrados. Outro fator que reforça a afirmação anterior é que os tratamentos com os níveis de arraçoamento de 13 e $16 \%$ do peso vivo dos animais sempre apresentaram sobra de ração no fundo das hapas, demonstrando que, nestes níveis, superava-se a quantidade de alimento para a saciedade do alevino. Portanto, pode-se afirmar que os dados de crescimento dos referidos trabalhos confirmam os deste experimento.

Além do efeito do nível de arraçoamento sobre os parâmetros de desempenho, o efeito desta variável pode ser notado na composição da carcaça dos peixes. Shimeno et al. (1997) cita o decréscimo da matéria seca e da gordura corporal da caraça de juvenis de carpa comum à medida que era diminuído o nível de arraçoamento. Portanto, um dos fatores importantes para a execução de trabalhos relacionados ao nível de arraçoamento é a análise da carcaça dos animais submetidos aos tratamentos.

O efeito quadrático dos parâmetros relacionados ao peso do alevino pode ser explicado, pois os níveis de arraçoamento inferiores ao ideal não possibilitaram ingestão do nível adequado de nutrientes para a expressão do máximo crescimento dos alevinos. Entretanto, os níveis de arraçoamento acima dos $10 \%$ do peso vivo ocasionaram diminuição do ganho de peso, efeito inesperado.

Uma explicação razoável para o decréscimo nos parâmetros de desempenho, nos níveis de arraçoamento acima dos $10 \%$, é que as sobras de ração nestes níveis acabavam sendo ingeridas em alguma extensão pelos peixes desses tratamentos. Essa sobra de ração, após algum tempo no fundo das hapas, além da perda de nutrientes solúveis, como vitaminas, sais minerais, entre outros, umedece e aumenta em volume e sua ingestão pode resultar em perda de apetite e diminuir o aporte de nutrientes, provocando redução do crescimento. 


\section{Conclusões}

O nível ideal de arraçoamento para alevinos de lambari (A. bimaculatus), sob temperatura média da água de $25^{\circ} \mathrm{C}$, é de $11,5 \%$ do peso vivo.

\section{Literatura Citada}

BOSCOLO, W.R.; HAYASHI, C.; MEURER, F. Digestibilidade aparente da energia e proteína bruta de alguns alimentos pela tilápia do Nilo (Oreochromis niloticus, L.). Revista Brasileira de Zootecnia, v.31, n.2, p.539-545, 2002.

FERNÁNDEZ, F.; MIQUEL, A.G.; GUINEA, J. Digestion and digestibility in gilthead sea bream (Sparus aurata): the effect of diet composition and ration size. Aquaculture, v.166, p.67-84, 1998 .

HAYASHI, C.; GALDIOLI, E.M.; NAGAE, M.Y. et al. Exigência de proteína para alevinos de lambari (Astyanax bimaculatus) (PISCES: CHARACIDAE). In: REUNIÃO ANUAL DA SOCIEDADE BRASILEIRA DE ZOOTECNIA, 36., 1999, Porto Alegre. Anais... Porto Alegre: Sociedade Brasileira de Zootecnia, 1999a. CD-ROM.

HAYASHI, C.; BOSCOLO, W.R.; SOARES, C.M. et al. Uso de diferentes graus de moagem dos ingredientes em dietas para tilápia do Nilo (Oreochromis niloticus $L$.) na fase de crescimento. Acta Scientiarum, v.21, n.3, p.733-737, 1999b.

HAYASHI, C.; MEURER, F.; BOSCOLO, W.R. et al. Frequência de arraçoamento para alevinos de lambari-do-rabo-amarelo (Astyanax bimaculatus). Revista Brasileira de Zootecnia, v.33, n.1, p.21-26, 2004.

HIDALGO, F.; ALLIOT, E.; THEBAULT, H. Influence of water temperature on food intake, food efficiency and gross composition of juvenile sea bass, Dicentrarchus labrax. Aquaculture, v.64, p.199-207, 1987.

JOHANSSON, L.; KIESSLING, A.; KIESSLING, K.H. Effects of altered ration levels on sensory characteristics, lipid content and fatty acid composition of rainbow trout (Oncorhynchus mykiss). Food Quality and Preference, v.11, p.247-254, 2000.

KUBITZA, F. Qualidade do alimento, qualidade da água e manejo alimentar na produção de peixes. In: SIMPÓSIO SOBRE MANEJO E NUTRIÇÃO DE PEIXES, 1., 1997, Piracicaba. Anais... Piracicaba: Colégio Brasileira de Nutrição Animal, 1997. p.63-116.

KUBITZA, F. Tilápia: tecnologia e planejamento na produção comercial. Jundiaí: Fernando Kubitza, 2000. 289p.

LIM, C. Practical feeding of tropical aquatic species. In: SIMPOSIO INTERNACIONAL SOBRE NUTRIÇÃO DE PEIXES E CRUSTÁCEOS, 1., 1995, Campos do Jordão. Anais... Campos do Jordão: Colégio Brasileiro de Nutrição Animal, 1995. p.159-171.
MEURER, F.; HAYASHI, C.; BOSCOLO, W.R. Digestibilidade aparente dos nutrientes e energia de alguns alimentos protéicos para juvenis de tilápia do Nilo (Oreochromis niloticus L.). Revista Brasileira de Zootecnia, v.32, n.6, p.1801-1809, 2003.

OSTRENSKY, A.; BOEGER, W. Piscicultura: fundamentos e técnicas de manejo. Guaíba: Agropecuária, 1998. 211p.

ROSTAGNO, H.S.; ALBINO, L.F.T.; DONZELE, J.L. et al. Tabelas brasileiras para aves e suínos: composição de alimentos e exigências nutricionais. Viçosa, MG: Universidade Federal de Viçosa, 2000. 141p.

SANTIAGO, C.B.; ALDABA, M.B.; REYES, O.S. Influence of feeding rate and diet from on growth and survival of Nile tilapia (Oreochromis niloticus) fry. Aquaculture, v.64, p.277-282, 1987.

SHIMENO, S.; SHIKATA, T; HOSOKAWA, H. et al. Metabolic response to feeding rates in common carp, Cyprinus carpio. Aquaculture, v.151, p.371-377, 1997.

SIPAÚBA-TAVARES, L.H.S. Limnologia aplicada à aqüicultura. Jaboticabal: FINEP, 1995. 70p.

SOARES, C.M.; HAYASHI, C.; GONÇALVES, G.S. et al. Substituição parcial da proteína da farinha de peixe pela de fontes protéicas alternativas em dietas para alevinos de lambari (Astyanax bimaculatus). In: REUNIÃO ANUAL DA SOCIEDADE BRASILEIRA DE ZOOTECNIA, 36., 1999, Porto Alegre. Anais... Porto Alegre: Sociedade Brasileira de Zootecnia, 1999. CD-ROM.

STOREBAKKEN, T.; AUSTRENG, E. Ration level for salmonids I. Growth, survival, body composition, and feed conversion in salmon fry and fingerlings. Aquaculture, v.60, p.189-206, 1987.

UNIVERSIDADE FEDERAL DE VIÇOSA - UFV. SAEG Sistema para análises estatísticas e genéticas. Versão 7.1. Viçosa, MG: 1997. 150p. (Manual do usuário).

VERBEETEN, B.E.; CARTER, C.G.; PURSER, G.J. The combined effect of feeding time and ration on growth performance and nitrogen metabolism of greenback flounder. Journal of Fish Biology, v.55, p.1328-1343, 1999.

VILELA, C; HAYASHI, C. Desenvolvimento de juvenis de lambari Astyanax bimaculatus (Linnaeus, 1758), sob diferentes densidades de estocagem em tanques-rede. Acta Scientiarum, v.23, n.2, p.491-496, 2001. 